\title{
Bridging the Gap between the Modeling Approach and the Experiment in Atom Probe Tomography
}

François Vurpillot ${ }^{1}$, Williams Lefebvre ${ }^{1}$, Lorenzo Rigutti ${ }^{1}$, Nicolas Rolland ${ }^{1}$, Florian Moyon ${ }^{1}$, Lorenzo Mancini $^{1}$, Hocine Hideur ${ }^{1}$, Didier Blavette ${ }^{1}$

1. Groupe de Physique des Matériaux, UMR CNRS 6634, Université et INSA de Rouen, Saint Etienne du Rouvray, France

Over the last twenty years, major breakthroughs in the development of Atom probe tomography (APT) make this nanoanalysis instrument an indispensable companion for the nano-scientist. For instance, complex nano-electronic device analyses are now routinely performed [1][2] with the introduction of laser pulsing [3][4], wide field of view atom probe and local electrode technology [7][8][9]. However, the standard tomographic reconstruction protocol remains very close to the original one developed at the early stage of the technique [10]. Even if attempts have been made to propose more evolved recipes, back projection onto a hemispherical specimen surface is still routinely used [8].

In a heterogeneous sample made up of several phases with different evaporation behaviors, the evaporation sequence is highly non uniform. It results in a non-hemispherical complex surface shape, which in turns gives rise to artefacts during reconstruction. Improving both the precision and the accuracy of Atom Probe Tomography reconstruction requires a correct understanding of this imaging process. In this aim, numerical modelling approaches have been developed for 15 years. The injected ingredients of these modelling tools are related to the basic physic of the field evaporation mechanism. The interplay between the sample nature and structure of the analyzed sample and the reconstructed image artefacts have pushed to gradually be more skeptical on the standard reconstruction protocol. The reconstruction artefacts have been first postulated and then demonstrated by numerical simulations, and they are now directly demonstrated experimentally by APT and TEM complementary analysis [2][8]. However, if great efforts have been made to understand the imaging process, at the moment, there are few theoretical frameworks to use the models in order to better reconstruct. The problem comes from first the difficulty to model the real structure of interest; the sample structure, the composition and its geometry are roughly known (this is the information sought by the nano-scientist). Second, the physical parameters injected in the model are also roughly known. Third, the model itself is complex, and time consuming.

In order to bridge the gap between the modelling approach and the experimental reconstruction protocol, several parallel ways must be followed. First improvements in the physical understanding of the emission process must be done, in order to refine the physical constants that are required and injected in the computation. A brief review of the models used in this aim will be presented. The modelling approach must also be improved, to be faster, and simpler, giving access to more straightforward parameters for the experimental point of view of the APT user. A pseudo analytic model was developed in this aim (fig. 1). In the current work, on comparison with numerical simulations, we validate the model and we highlight the origin of some field evaporation features. Finally, this paper will show the advantages to use combined characterization techniques to improve our knowledge of the sample before, after or during the imaging process in APT. Characterization of the same object with other techniques such as TEM tomography, and micro-photoluminescence proved to be essential to make the link between the model and the reconstructed image. 
References:

[1] A. K. Kambham, et al., Ultramicroscopy, vol. 111, no. 6, pp. 535-539, May 2011.

[2] A. Grenier, et al.,” Ultramicroscopy, vol. 136, pp. 185-192, Jan. 2014.

[3] B. Gault, et al.” Rev. Sci. Instrum., vol. 77, no. 4, p. 043705, Apr. 2006.

[4] J. H. Bunton, et al.,” Microsc. Microanal., vol. 13, no. 06, pp. 418-427, 2007.

[7] T. F. Kelly et al. Microsc. Microanal. Off. J. Microsc. Soc. Am. Microbeam Anal. Soc. Microsc. Soc. Can., vol. 10, no. 3, pp. 373-383, Jun. 2004.

[8] D. J. Larson et al., Local Electrode Atom Probe Tomography: A User's Guide. New York: Springer, 2013.

[9] M. K. Miller and R. G. Forbes, Atom-Probe Tomography: The Local Electrode Atom Probe. New York: Springer-Verlag New York Inc., 2014.

[10] P. Bas et al.” Appl. Surf. Sci., vol. 87-88, pp. 298-304, Mar. 1995.

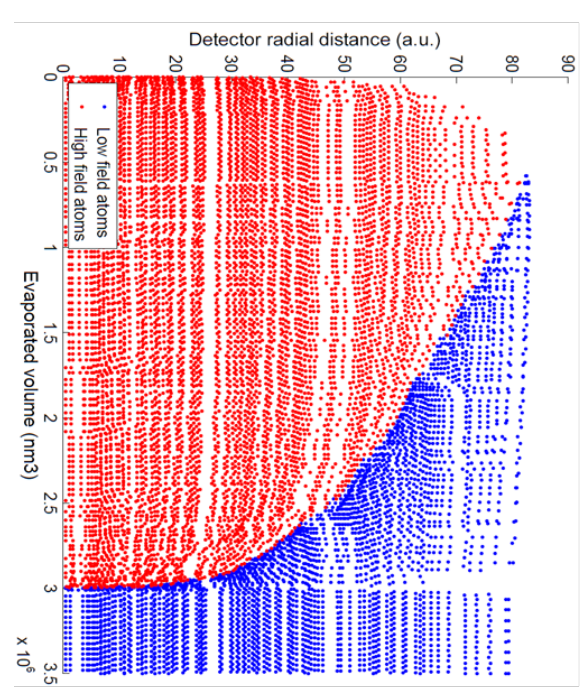

(a)

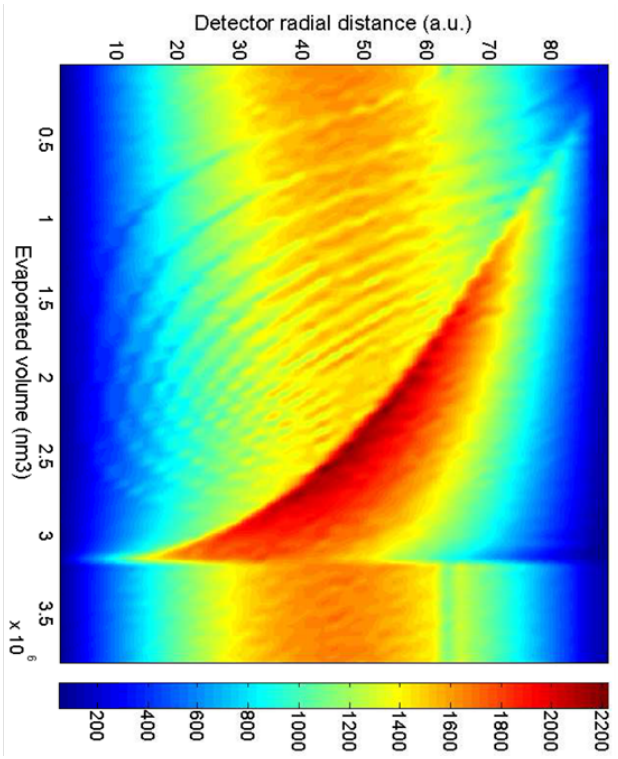

(b)

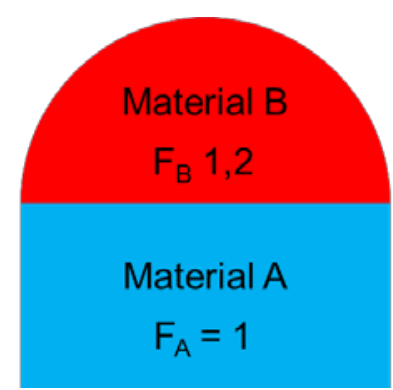

(c)

Figure 1. Comparison of the radial density of impacts in function of the analyzed depth obtained by simulating the evaporation of a layer $B$ with high evaporation field $\left(E_{B}=1.2 E_{A}\right)$ deposited on a low evaporation field planar substrate A with (a) an atomistic simulation of the evaporation (b) a faster analytic simulation. (c) Schematic drawing of the tip modelled in both cases. 